\title{
Health Care Systems Engineering
}

\author{
Jingshan Li ${ }^{1}$ Andrea Matta ${ }^{2}$ Evren Sahin ${ }^{3}$
}

Published online: 12 December 2015

(C) Springer Science+Business Media New York 2015

Cost containment in health care services is becoming more and more important in a context in which the demand for health care services is increasing because of the population ageing (from $4 \%$ in 2010 to nearly $10 \%$ in 2050 in OECD countries), the increase in chronic pathologies and the social changes over recent decades. The way in which health care service organizations are managed affects their global system performance and needs to be re-designed in order to address the requirements of the new environment.

The primary objective of this Special Issue is to publish papers that reflect recent developments to improve the service delivery in health care systems. Scientists and practitioners presented their current research topics at the International Conference on Health Care Systems Engineering (HCSE) 2013 held in Milano, Italy, at San Raffaele Hospital in the areas of health care systems modeling, simulation, and optimization. All of the contributions selected in this Special Issue propose novel methods, models and analyses illustrated by real world examples.

Andrea Matta

matta@sjtu.edu.cn

Jingshan Li

jingshan@engr.wisc.edu

Evren Sahin

evren.sahin@centralesupelec.fr

1 Department of Industrial and Systems Engineering, University of Wisconsin-Madison, 1513 University Avenue, Madison, WI, USA

2 Department of Industrial Engineering and Management, Shanghai Jiao Tong University, 800 Dong Chuan Road, Shanghai, China

3 Laboratoire Genie Industriel, CentraleSupélec, Université Paris-Saclay, Grande Voie des Vignes, 92290 Chatenay-Malabry, France 
For this Special Issue fourteen papers have been selected for publication after a thorough peer-review according to the standards of the FSM journal. Six of these papers have been previously presented at HCSE 2013 and significantly extended for this Special Issue. The other eight papers come from different channels.

\section{Papers in the Special Issue}

The first paper by Helber, Böhme, Oucherif, Lagershausen and Kasper presents a hierarchical layout planning approach that determines the locations of departments and wards of a hospital, in order to reduce the inter-department transportation costs. They apply their approach to a fictitious system of connected hospital buildings, where they determine the allocation of the organizational units to minimize the consumption of resources used in transportation processes.

Hulshof, Mes, Boucherie and Hans develop a stochastic approach for a tactical healthcare planning problem, i.e. elective patient admission planning and allocation of hospital resource capacities, by modelling it as a (Approximate) Dynamic Programming problem (ADP), for large, real-life sized instances encountered in Dutch hospitals. Their solution approach combines various techniques within the ADP-framework and the field of mathematical programming. The performance of this approach is assessed by comparing it to two "greedy" planning approaches. Results obtained indicate that the algorithm performs better than the greedy approaches, in reasonable run times.

The paper by Mallor, Azcárate and Barado deals with bed-management policies that aim at minimizing patient rejections or minimizing the length of stay shortening by modifying the service time rates of servers. The authors propose either analytical or numerical solutions obtained via simulation-optimization depending on the specific formulation. The approach is general enough to be applied to the majority of wards or hospitals.

The paper by Zhong, Song, Li, Ertl and Fiedler introduces a Markov chain model for design and analysis of a new Gastroenterology (GI) clinic at Digestive Health Centre of University of Wisconsin Health. The model is extended to nonMarkovian scenario and validated through observed data. Using the model, various design options under different system variations are investigated, which provide recommendations for the new GI clinic.

The next paper by Kuo, Rado, Lupia, Leung and Graham presents a simulation case study to analyze patient flow in a hospital emergency department. By integrating with meta-heuristics, a simulation-optimization approach is used to obtain a good set of estimate of input parameters even with incomplete data. Then the impact of possible changes are evaluated to provide decision support for operations managers.

The paper by Thorwarth, Rashwan and Arisha deals with the problem of developing simple but accurate enough representations of complex discrete event systems that can easily managed by practitioners in health care systems. The authors show how to derive an analytical model for the estimation of the staff utilization levels in Emergency Departments using output collected from simulation 
experiments. The analytical model has in the simplicity its strength as it can be used as decision making tool for staffing related problems.

Guo, Wu, Li, Song and Rong study integration of elective surgery and surgical nurse scheduling. An integer programming model is proposed to schedule elective surgeries and surgical nurses simultaneously. Based on this, an efficient genetic algorithm is used to ease computational complexity. A case study is presented to validate the performance and compare with the traditional two-stage approach which sequentially schedules surgeries and nurses.

The paper by Visintin, Cappanera and Banditori deals with the classical master surgical scheduling problem in hospitals. The major contribution of this work is to investigate the impact, in terms of scheduled surgeries, of the flexible management of three critical resources, namely surgical teams, operating rooms and surgical units. The main result is that, in order to maximise the number of surgeries scheduled, it is sufficient to introduce flexibility with respect to surgical teams and operating rooms. The work is based on real data from a children's hospital in Italy.

The paper by Addis, Carello, Grosso and Tànfani proposes a robust optimization approach that selects a set of patients among available elective patients and assigns them to a set of available operating rooms. They consider two types of uncertainty within the planning horizon: new patient arrivals and surgery times (that may be longer than expected). At the beginning of each week, a time window of several weeks is scheduled, and the first week solution is applied; the schedule for the other weeks is kept as a reference for the next iterations. Each schedule is produced by solving an ILP optimization model. Computational tests performed with probability distributions derived from real-life data offer promising results.

The paper by Lee and Shin deals with the problem of early detecting influenza outbreaks. They propose a particle filtering method to combine immediatelyavailable-but-less reliable syndromic surveillance data with reliable-but-timedelayed influenza-like-illness report data. The numerical results show that pandemic scenario state can be better estimated by utilizing both data sets than when using only one of them. The amount of improvement depends on relative credibility and length of delay in influenza-like-illness data.

The paper by Argiento, Guglielmi, Lanzarone and Nawajah proposes a Bayesian framework to study home care networks with complex structures and high randomness. The patients' demand evolution is described by means of a generalized linear mixed model, and a Markov chain Monte Carlo simulation model is used to obtain posterior densities of the parameters. A real case study is introduced to illustrate the applicability and validity of the method.

Redjem and Marcon study the routing and scheduling problems in home care services. A heuristic approach, the Caregivers Routing Heuristic (CRH), is developed and evaluated with real size instances. The numerical results show that the $\mathrm{CRH}$ is computationally efficient and less sensitive than the exact approaches in both complexity axes, the temporal dependencies constraints and the ratio of the number of care activities per caregiver.

The paper by Yalçındağ, Matta, Şahin and Shanthikumar aims at providing good travel time estimates that would be used when assigning care givers to home 
healthcare patients. Their method, based on the Kernel Regression (KR) technique, enables the empirical modelling of care givers' travel routes. Numerical results based on realistic problem instances indicate that it performs better than the average value and k-nearest neighbor search methods. The use of the KR technique offers promising perspectives to home care operations by offering the possibility to develop new assignment techniques that would first assign patients to care givers and then define their routes.

The last paper is by Rauner, Niessner, Leopold-Wildburger, Peric and Herdlicka, who introduce a discrete-event-based policy simulator that can be used as a training tool for medical emergency staff, in case of mass casualty incidents (MCI). In particular, the developed model enables to learn the complex processes of a rescue operation after an MCI and helps to analyze different policies in terms of vehicle and patient scheduling, staff and material planning on site. The authors also conduct an experimental study with 96 participants including students, health care practitioners and researchers to investigate the potential of this tool and to assess the learning behaviour of participants during the game.

\section{Concluding remarks}

This Special Issue has greatly benefited from the cooperation among the authors, reviewers, and editors. We would like to express our sincere thanks to the reviewers for their excellent and timely refereeing. Last, but not least, we thank all authors for their contributions which made this Special Issue possible. 